\title{
AOR
}

Selected Papers of \#AolR2020:

The 22nd Annual Conference of the

Association of Internet Researchers

Virtual Event / 13-16 Oct 2021

\section{PLAYING AT THE POLLS: VIDEO GAMES IN/AS PLATFORMS OF POLITICAL PARTICIPATION}

\author{
Christine H. Tran \\ University of Toronto \\ Bonnie "Bo" Ruberg \\ University of California Irvine \\ Daniel Lark \\ University of Southern California \\ Nicholas-Brie Guarriello \\ University of Minnesota
}

\section{Panel Rationale}

In 2020, video games and livestreaming came to the fore of American politics as COVID-19 disrupted social and economic life in the United States. Politicians like Joe Biden and Alexandra Ocasio-Cortez turned to games like Animal Crossing and streaming platforms like Twitch as new media vehicles for political messaging, including a Biden-themed Animal Crossing island, a Biden-Harris "No Malarkey Station" in Fortnite, Get Out the Vote livestreams, and virtual town halls featuring politicians and microcelebrity streamers. Historically, games have operated in US party politics as figurative platforms in the form of "talking points" on media violence, youth welfare, and censorship. Throughout 2020, however, video games and livestreaming were refigured as computational infrastructures through which American politicians may connect with (often young) constituents. This panel explores the rise of ludic technologies as both rhetorical and locative "platforms" for American political participation, from livestreaming to online multiplayer game spaces and beyond.

Suggested Citation (APA): Tran, C. H., Ruberg, B., Lark, D., Guarriello, N.-B.. (2021, October). Playing at the polls: Video games in/as platforms for political participation. Panel presented at AolR 2021: The 22nd Annual Conference of the Association of Internet Researchers Virtual Event: AolR. Retrieved from http://spir.aoir.org. 
As we demonstrate, these shifts are not without controversy or complaint. Platforms for game playing and sharing, like Twitch, have struggled to manage their public image as community stewards and moderators amidst rising expectations that platform and technology companies recognize, react, and respond to racial and social justice issues. For example, on 5 July 2020, Amazon's \$15 billion (USD) game streaming platform Twitch concluded its LGBTQ Pride Month celebrations with a video spotlighting queer creators declaring, "The G In LGTBQ+ Also Stands for Gamer!" Queer users and commentators quickly archived and denounced Twitch's ahistorical publicity move as "another identity-related L[oss]" taken by the world's largest game streaming platform (Stephen, 2020).

Far from neutralizing or infantilizing politics, games further embed public policy into the platformed systems of content moderation, community management, and governmental and economic power of software companies over American sociality (Gorwa, 2019; van Dijck, 2013). Whereas quips like Hillary Clinton's "Pokémon Go to the Polls!" became ironic media gaffes and memes in the 2016 Presidential election, ludic interfaces today are promising direct and adhesive engagement between politicians and populace, albeit along precarious lines.

Games scholarship is well-positioned to address the technological and social debates of U.S. political discourse. Within the study of game subcultures, their players and spaces, identities and their politics have been figured as technological issues (Gray 2020; Ruberg 2019). Bolstered by this scholarship, we focus here on the social effects and affordances of games upon classed and gendered identities. We attend to the mass repoliticization of games and question the politics of identity, content moderation, and labour that are downloaded into policy when party communication becomes playful.

Encompassing a broad collection of interdisciplinary research (political economy, queer studies, media and communication studies, feminist science and technology studies) from scholars working across North America, this panel engages with the empirical and theoretical questions raised by the tactical use of ludic spaces as content delivery systems for policy, polling, and political participation.

Our first paper examines how Twitch streamers redefine "virtual picket lines" in alternative labour movements against one of the world's largest platform technology employers, Amazon. The second paper explores the queer political imaginations of islandic virtual spaces in Nintendo's Animal Crossing from Biden Island to beyond. The third paper explores the game Alexandria-Ocasio Cortez's livestreaming as a key transformation in the sociotechnical infrastructures of U.S. political publicity. Finally, our fourth paper analyzes Twitch's revamped content moderation policy strategies in the aftermath of users reacting to the January 2021 Capitol Hill insurrection on the livestreaming platform.

To summarize, we call for deeper cultural-level critique and historicization of the ways games have enabled porosity between social, recreational, and civic interfaces. Games are "future-oriented commodities" that "sell dreams" (Bulut, 2020, p. 22) due to their ideations of agentic and rules-driven relationships between self/other, self/society. Far from offering a simple imagination of independence from the physical limitations of 
traditional political communication networks, this frenetic interplaying from political campaigns signals the widespread integration of platforms and their physical, governmental, and economic infrastructures into social life (Nieborg and Poell, 2018). As we gamify politics, who is left without a console?

\title{
References
}

Bulut, E. (2020). A precarious game: The illusion of dream jobs in the video game industry. Cornell University Press.

Gorwa, R. (2019). What is platform governance? Information, Communication and Society, 22(6), 854-871.

Gray, K.L. (2020). Intersectional tech: Black users in digital gaming. Louisiana State University Pres

Nieborg, D. B., \& Poell, T. (2018). The platformization of cultural production: Theorizing the contingent cultural commodity. New Media \& Society, 20(11), 4275-4292.

Ruberg, B. (2019). Video games have always been queer. New York University Press.

Stephen, B. (2020, September 15). Twitch takes another identity-related L. The Verge. https://www.theverge.com/2020/9/15/21438659/twitch-hispanic-heritage-monthblack-lives-matter-lgbt-pride-month

van Dijck, J. (2013). The culture of connectivity: A critical history of social media. Oxford University Press.

\section{DON'T CROSS THE STREAMS: TWITCH, NETWORKED SOLIDARITY \& AMAZON'S VIRTUAL PICKET LINE}

\author{
Christine H. Tran \\ University of Toronto
}

\section{Introduction: To Stream or Scab?}

"NO SCABS, NO TWITCH, NO AMAZON," barks a comment on United States senator Bernie Sanders' debut livestream on the livestreaming platform Twitch.tv (Grayson, 2019, para. 11). On 10 July 2019, the Democratic politician made his inaugural livecast on the Amazon-owned platform popular for video game broadcasting and spectatorship. Audiences and commentators noted, however,that Sander's virtual town hall launch apparently "crossed the picket line" by broadcasting on Amazon's "Prime Day." In recent years, the annual promotional sales "holiday" has been a scheduling point for Amazon workers to organize labour stoppages in protest of their precarious work conditions and low wages across the conglomerate's global supply chain. Many Twitch streamers also chose to "blackout" their channels on Prime Day, in defiance of contracted broadcast schedules, in order to signal their solidarity with fellow Amazon workers. 
Despite Sanders' record of allyship with alternative labour movements, his Twitch presence as workers boycott Amazon platforms was seen as a strikebreaking transgression. As one commenter echoed: "You crossed [the] picket line you are going to lose subscriber [sic]" (para. 10). Responding to the hyper pervasiveness of digital platforms across the working and leisure lives of Amazon's employees and consumers, this paper is driven by three primary questions: What is a picket line to a video game livestreamer? What solidarities, if any, do Twitch microcelebrities extend to other Amazon dependents? How do livestreaming labour link Amazon's workforces into platform dependency?

Guided by critical technocultural discourse analysis (CTDA) of Twitch streamer's social media responses to Amazon work stoppages from 2018 to 2021, I explore the technocultural creation of the "virtual picket line" in the platform's ecosystem of Twitch. I review emerging cultural narratives on streamer boycotts, and Amazon labour stoppages during Prime Days, and how Twitch streamers use information technologies, like hashtags, to demarcate acceptable strike behaviors and broadcast their social alignment with Amazon workers. I locate the vocational figure of the Twitch "livestreamer" (and more broadly, the digital influencer) in an ambivalent yet autonomous relation to the means of labour messaging that is livestreaming along the picket line.

\section{Contexts \& Theory}

Since its $\$ 1$ billion acquisition by Amazon in 2014, Twitch.tv has risen as a bedrock of social media phenomena in mainstreaming of game community influencers. Recent studies into the working lives of Twitch streamers have noted the importance of selfstartership and neoliberal work ethics to their creator culture (Guarriello, 2019; Johnson and Woodcock, 2019). This scholarship has predominantly positioned Twitch's workforce in the history of game player cultures emerging around the platform (Consalvo, 2017; Taylor, 2018), but its business evolution has also been positioned as a driver of asymmetries and dependencies between autonomous users and Amazon technology (Partin, 2020). Less attention has been directed to Twitch's cultural and technical heritage as labour organizing. Counter to the imagination of streamers as selffocused entrepreneurs--bolstered by the meritocracy endemic to gamer culture--recent headlines show streamers how can and do critique their parent company's policies from a collective consciousness.

In February 2021, for example, Twitch's streamers successfully advocated against Amazon's imposition of anti-union advertising in their broadcasts. Amazon removed the video advertisements off several streams (Clark, 2021). In 2018, anonymously run channels like "Scabstream" livecast publicized TwitchCon's crossing the picket line at the Marriott Hotel, where staff had striked against low wages and the implementation of Amazon technologies in their workplaces while the Amazon-Twitch toasted its top streamers on the same workgrounds (Malgrem, 2019).

Twitch streamers' publicization of internal strike action characterizes what Brophy et. al (2015) term "networked solidarity," where the labour messaging between cultural workers and precarity is ambivalently negotiated with ICT technologies. The same 
platforms that extend the site of precarity for workers--from streamers to manual labourers subject to Amazon's technological supremacy--also forms sites of resistance and counter-publicity. From these technical practices, I explore how workers move, merge, and manifest their "picket lines" against (and for) Amazon's politics.

\section{Method}

Using Andre Brock Jr.'s (2018) method of Critical Technocultural Discourse Analysis (CTDA), I shall review key instances of Twitch streamer resistance to Amazon's employment policies, especially at times of labour stoppages across the supply chain, using online tools: Twitter, online forums, weblogs, Reddit, and direct communications that have been dated from 2018 to 2021.

I direct my CTDA to Twitch streamers' use of \#TwitchBlackOut and \#NoTwitchScabs hashtags in organizing labour stoppages. As a multimodal technique, a CTDA holistically integrates disparate data points across an ecosystem: tweets, channel details, messages, and headlines. I do a close-reading of these texts as interfaces between streamers, other Amazon workers, and the public as continuous conversations. In accordance with CTDA's requirement that "[t]echnocultural discourses [...] be framed from the cultural perspectives of the user AND of the designer" (p. 1020), I focus on the expressed autonomy of streamers as designers of their own employee identity, even as they are mutually shaped by the interfaces of Amazon-owned livestreaming.

\section{Contributions}

Despite lingering views that influencing and content creator labour is a site of frivolity, the cultural work of livestreamers on the Amazon picket line offer an alternative pathways for digital organizing. This paper contributes to the growing study of labour collectivizing and cultural work on platforms (Cohen and de Peuter, 2020; de Peuter, 2014). While aspirations of Twitch streamers unionize amongst themselves remains aspirational (D'Anastasio, 2020), the survival of self-criticism organized stream stoppage--directed outward for other Amazon employees highlights Twitch's workforce as a producer of of "work-generated content" (Qiu, 2016) against a technology giant. As game-centric and livestreaming platforms become widespread into other areas of social life, the Twitch picket line represents a pivotal site of struggle for the future of digital labour and alternative pathways therein.

\section{References}

D'Anastasio, C. (2020, July 8). Is it game streaming's turn for a labor revolution? Wired. https://www.wired.com/story/mixer-livestreaming-labor-rights/

Brock, A. (2018). Critical technocultural discourse analysis. New Media \& Society, 20(3), 1012-1030.

Brophy, E., Cohen, N. S., \& de Peuter, G. (2015). Labor messaging practices of autonomous communication. In R. Maxwell (Ed.), The Routledge Companion to Labor and Media (pp. 315-326). Routledge. 
Clark, M. (2021, February 25). Twitch, owned by Amazon, pulls Amazon's anti-union ads. The Verge. https://www.theverge.com/2021/2/25/22301352/twitch-removesamazon-anti-union-ads

Cohen, N. S., \& de Peuter, G. (2020). New Media Unions: Organizing Digital Journalists. Routledge.

Consalvo, M. (2017). When paratexts become texts: de-centering the game-as-text. Critical Studies in Media Communication, 34(2), 177-183.

Grayson, N. (2019, July 17). Bernie Sanders Finally Appears On His Own Twitch Channel-During Amazon Boycott. Kotaku. https://kotaku.com/bernie-sandersfinally-appears-on-his-own-twitch-channe-1836459495

Guarriello, N.-B. (2019). Never give up, never surrender: Game live streaming, neoliberal work, and personalized media economies. New Media \& Society, 21(8), 1750-1769.

Johnson, M. R., \& Woodcock, J. (2019). "It"s like the gold rush': the lives and careers of professional video game streamers on Twitch.tv. Information, Communication and Society, 22(3), 336-351.

Malmgren, E. (2018, November 2). Twitch crosses the picket line. The Nation. https://www.thenation.com/article/archive/twitch-amazon-videogames-hotelpicket/

Partin, W. C. (2020). Bit by (Twitch) bit: "Platform capture" and the evolution of digital platforms. Social Media + Society, 6(3), 2056305120933981.

de Peuter, G. (2014). Beyond the model worker: Surveying a creative precariat. Culture Unbound, 6(1), 263-284.

Qiu, J. (2016). Social media on the picket line. Media Culture \& Society, 38(4), 619633.

2. POLITICAL ISLANDS, QUEER ISLANDS: COMPARING CULTURAL NARRATIVES AROUND SECOND LIFE AND ANIMAL CROSSING: NEW HORIZONS

Bonnie "Bo" Ruberg

University of California Irvine

\section{Virtual Islands Present and Past}

The video game Animal Crossing: New Horizons (Nintendo) was released on March 20, 2020 , roughly one week after much of the United States went into lockdown in response to COVID-19. Already hotly awaited by fans of the Animal Crossing series, New Horizons became a veritable phenomenon in the early days of the pandemic, when it garnered both millions of players and a seemingly unending stream of news media coverage. These articles, which placed New Horizons at the center of broader 
discussions about digital media in the time of COVID-19, often told uplifting human interest stories--participating in a moment when, as Rainforest Scully-Blaker and Emily Flynn-Jones have written, "suddenly, a game about purchasing a 'Deserted Island Getaway Package'... took on new significance in popular culture and regimes of selfcare" (2020, p. 5). Of particular interest in many reports were New Horizons' islands: unique locales, cultivated either by individuals or organizations, that players could visit in-game. Among New Horizons' many publicly-accessible islands, two types seemingly deemed most newsworthy were those that promoted specific political figures, such as then presidential candidate Joe Biden (whose island was later removed), and those that held space for LGBTQ communities (e.g. Maurice, 2020).

While the buzz surrounding New Horizons presented these islands as new--a shift in how both politics and activism were being done in digital spaces--a longer view of internet history reveals that this was, in fact, the second time that online islands, one of many aquatic metaphors of the internet (Thibault, 2015), became the stuff of public interest. This research compares the cultural narratives surrounding New Horizons in 2020 with those that surrounded the virtual world Second Life more than a decade earlier, between roughly 2006 and 2008. Second Life differs considerably from New Horizons in its design and affordances, yet the two share that they are structured spatially around user-cultivated islands. Like New Horizons after it, Second Life was the subject of a media flurry, sparked when corporations and institutions of higher learning began building islands in the game (e.g. Girard, 2006). Long before the lockdowns of COVID-19, which brought with them a sense that internet users were themselves islands scattered at sea, Second Life's islands were also raising now-familiar questions about what happens when spaces of online play become spaces for public messaging.

\section{Methods: Media Traces of Cultural Imaginaries}

To establish a rich understanding of the rhetorics that have characterized public discussions of islands in Animal Crossing: New Horizons during the COVID-19 pandemic, as well as those parallel discourses that formed around Second Life's islands in the early 2000s, this work draws from a collection of articles from online and print news sources. These articles have been assembled using a variety of search tools and archives and have been selected to represent both the breadth and depth of reporting on these topics. Articles considered for this work originally appeared in venues oriented either toward a mainstream readership or toward readers interested in video games and tech culture. After assembling this collection of articles, the author has performed a qualitative analysis of the texts, approaching them through a critical cultural lens and coding them according to repeated themes. This has allowed the author to simultaneously trace patterns across texts and to identify notable differences. Such methods are not intended to be comprehensive; they are also limited by their focus on news reporting, which does not capture, for example, player experiences with New Horizons and Second Life respectively. What these methods offer, however, is the opportunity to map traces of broader cultural imaginaries, using media to track ephemeral, unspoken attitudes toward life online.

\section{Changing Seas of LGBTQ Issues in Video Games}


Comparing the cultural narratives that have emerged around Animal Crossing: New Horizons and Second Life reveals both striking similarities and telling differences. To an extent, this comparison helpfully historicizes today's discussion of how politics, as well as other professional arenas, are entering digital games. Such conversations, it turns out, are structured around rhetorics of newness and yet themselves repeat. This research finds, for instance, that news articles that respond with curious bemusement to the presence of the Biden-Harris island in New Horizons are matched by a parallel set of articles from an earlier moment expressing the same incredulity about the creation of areas in Second Life like American Apparel's corporate island or the construction of an island by the University of Wisconsin. Across these texts, the image of the island continues to hold sway, representing (not unproblematically) the "exoticness" that new virtual spaces are imagined to offer for the neoliberal, colonial digital subjects.

At the same time, this comparison reveals how these conversations about online islands have always been more about fantasies of digital life than their realities. Articles about New Horizons tend to oversell the importance of its islands, portraying them as grandscale meeting places when they are, in truth, little more than publicity stunts that cannot hold more than eight players at a time. Earlier articles from the height of public interest in Second Life demonstrate a similar pattern: the most talked-about islands, now almost defunct and abandoned, were already largely empty and underutilized in their heyday. Thus, it is not only the phenomenon of the virtual island that repeats, but also the ways that broader cultural narratives attempt to make sense of (and, in doing so, contort) the cultural meaning of these islands.

However, stark difference surfaces in the ways that news reporting on New Horizons and Second Life discuss issues of gender and sexuality, drawing attention to shifts in the imagined relationship between digital spaces as political and digital spaces as queer. Articles about New Horizons often highlighted islands dedicated to LGBTQ activism, such as the so-called "Pride Island," looping these islands into larger stories about the exciting capacity of the game to create safe spaces for self-expression, both personal and political. Articles about Second Life, by contrast, largely derided the world for its association with sexual subcultures, including LGBTQ ones. This evidences a shift in the cultural landscape around video games. Conversations about in-game islands may largely remain unchanged, but attitudes toward the place of LGBTQ people in those games surely have. Whereas, previously, the presence of queer content in Second Life was deployed to delegitimize the game, casting it as a cesspool of depravity, present-day stories about New Horizons use queerness to legitimize the game and its cultural value. Thus, these stories document a shift not only in how queer people are perceived but also how they are instrumentalized to tell specific cultural narratives.

\section{References}

Flynn-Jones, E., \& Scully-Blaker R. (2020). Foreword: To all the animals we've crossed before." Loading... The Journal of the Canadian Game Studies Association, 13(22), 1-6. 
Girard, N. (2006, June 16). Apparel brand gets 'Second Life." CNet. https://www.cnet.com/news/apparel-brand-gets-second-life/.

Maurice, E. P. (2020, June 16). 'Pride Island' coming to Animal Crossing, with rainbow march and queer club in support of Black Lives Matter. Pink News.

https://www.pinknews.co.uk/2020/06/16/animal-crossing-global-pride-island-weare-social-singapore-black-lives-matter-nintendo/.

Thibault, G. (2015). Streaming: A media hydrography of televisual flows. View: Journal of European Television History and Culture, 4(7), 110-119.

\title{
3. THEY STREAM AMONG US: POLITICAL LIVESTREAMING DURING THE 2020
}

\section{U.S. ELECTION}

\author{
Daniel Lark \\ University of Southern California
}

\section{Background}

This paper provides a platform, discourse, and textual analysis of political livestreams, focusing on the use of Twitch by Democratic Party politicians Alexandria Ocasio-Cortez and IIhan Omar during the 2020 election cycle and Covid-19 pandemic in the United States. I argue that livestreaming platforms like Twitch.tv are becoming sites not just for the delivery of political messaging and content, but infrastructure for interaction with political constituents and citizens. Political streaming blends together the technological, cultural, and affective practices of video game livestreaming, establishing connections with already popular streamers and taking advantage of the platform's ability to generate intimacy and familiarity with audiences in order to drive users towards participation in electoral politics. While existing scholarly literature has explored video game live streaming cultures and the practices of amateur and professional streamers (Taylor, 2018) or the integration of social media platforms like Facebook into political campaigns (Kreiss and McGregor, 2017), not much scholarship has described the use of livestreaming platforms by politicians or explored the relationship between video game live streaming and existing political institutions in particular.

As part of a complex sociotechnical system, political livestreaming raises important questions about transformations in politics during contentious periods and the ownership of increasingly central public sphere platforms by private corporations. Platforms are increasingly recognized as having important public, infrastructural functions that must be interpreted and possibly protected from abuse or misuse (Plantin et al, 2016). As infrastructure, platforms also create strong social and affective attachments that go beyond their base technical functions, structuring forms of political rationality, fantasy, and desire (Larkin, 2013). Platforms increasingly blend together social, political, and economic logics into highly expressive communicative acts well suited to neoliberal political structures (Nieborg and Poell, 2018; van Dijck et al., 2018).

\section{Political Livestreaming - A Case Study}


During the election cycle, Ocasio-Cortez streamed a live session of her playing the game Among Us (2018) with other members of "the Squad," the popular name for the small group of young, progressive, and social media savvy Democrats who have been elected to Congress in 2018, and several prominent Twitch streamers, including @HasanAbi, a former journalist turned streamer, and @Pokimane, one of the most popular streamers on the platform. During the stream, Ocasio-Cortez, Omar, and the streamers discussed the 2020 election, get out the vote initiatives, and progressive politics in between rounds of play. While AOC is not the first politician to combine video games with political advertising, she is among the first politicians to engage with games and streaming culture on their own terms. These events reflect an engagement with the cultures and practices of users and communities. Livestreaming platforms like Twitch are not just mere delivery vehicles for political messaging that work alongside traditional engagement practices like rallies, mailers, canvassing, and town halls, but complex systems that transform message and messaging itself.

As novice gamers, Ocasio-Cortez and Omar were not adept at playing the game, were unused to the technological demands of live streaming, and were unfamiliar with some of the cultural practices and nuances of engaging with the audience. This situation, however, did not damage their credibility or reception. In fact, it seemed to have the opposite effect, lending their stream a sense of authenticity and charm to which viewers responded positively. Further, the decision to play Among Us is also significant. The game became quite popular during the spring and summer of 2020 as a form of entertainment during periods of social distancing and minimal in-person interaction due to the spread of Covid-19. Yet, popularity is not the only significant factor. Among Us can be played across multiple platforms and consoles, including mobile phones, making it widely available to many users for a very low cost (about \$5 USD). Because it is multiplatform, the game is not computationally and graphically demanding and can be played on machines with little memory or without high quality graphics cards. The game's structure is adapted from familiar playground games, with simple gameplay that is relatively easy to learn, and where successful play does not rely on technical skill or speed, making it a game for novice and expert players alike. Gameplay has multiple periods of breaks and silences, which allow many opportunities for the streamers to chat with the audience about the game or politics. As such, Among Us was a highly strategic game choice that brought together viewers across multiple platforms, points of access, and skill levels and allowed for multiple points of communication with the audience.

As a multiplayer social game about social trust and collective decision making, Among Us draws direct parallels between the game and the broader political context of the United States, where trust in political institutions is rapidly decreasing and a significant portion of the population does not participate in electoral politics. Among Us is an ostensibly democratic game, where players are tasked with fixing their spaceship before an impostor among them completely sabotages their efforts. While the impostor tries to kill the crew of the ship, all players can meet, deliberate, and finally vote on who they think the impostor is and whether they should remove the suspect from play. If they correctly identify and vote out the impostor, the other plays win. Successful play is not just about completing one's assigned spaceship maintenance tasks, but about paying 
careful attention to your other crew members and trying to discern who is just pretending to work while actively sabotaging the ship.

\section{Analysis}

By playing Among Us together, AOC, Omar, and the streamers play a game that eerily reflects existing political structures while simultaneously encouraging citizens' engagement with and trust in traditional electoral politics. I argue that their amateur performance of gameplay and Twitch streaming aligns well with aspects of games streaming culture on Twitch. Performing poorly and being a bit unprepared for technical difficulties, while creating some problems, also created personal identification and narrative excitement, where overcoming technical problems becomes a source of humor and initiation into the routine challenges of livestreaming. Among Us can therefore be read as a mirror of the U.S. neoliberal political situation, where individual members of the public are increasingly tasked with the responsibility for maintaining their social, economic, and political infrastructures, inundated with potentially fake information and news, and potentially surrounded by bad actors who take advantage of processes of collective decision making and social uncertainty to shift society's trajectory towards their own political destinations.

\section{References}

Kreiss, D., \& McGregor, S. (2017). Technology firms shape political communication: The work of microsoft, Facebook, Twitter, and Google with campaigns during the 2016 U.S. Presidential Cycle. Political Communication, 35(2), 155-177. https://doi.org/10.1080/10584609.2017.1364814

Larkin, B. (2013). The politics and poetics of infrastructure. Annual Review of Anthropology, 42(1), 327-343. https://doi.org/10.1146/annurev-anthro-092412155522

Nieborg, D. B., \& Poell, T. (2018). The platformization of cultural production: Theorizing the contingent cultural commodity. New Media \& Society, 20(11), 4275-4292. https://doi.org/10.1177\%2F1461444818769694

Taylor, T. L. (2018). Watch me play: Twitch and the rise of game live streaming. Princeton University Press.

van Dijck, J., Poell, T., \& de Waal, M. (2018). The Platform society: Public values in a connected World. Oxford University Press.

\section{POGLITICALLY CORRECT: CONTENT MODERATION, OFF-PLATFORM BEHAVIOR, AND EMOTES ON TWITCH}

Nicholas-Brie Guarriello

University of Minnesota

You're my little PogChamp, right? 
On January 6, 2021 white supremacists led an insurrection to ultimately overturn the 2020 United States Presidential Election results. After Ryan "Gootecks" Guiterrez incited further violence at Capitol Hill via his Twitter account, Twitch removed the popular community wide PogChamp emote that contained an image of his surprised, wide-eyed and open-mouthed face. The banning of this globally used emote to signpost a moment of excitement or surprise in a livestream on Twitch had two impacts: 1) users of the platform took to Reddit, Discord, and Twitter to express discontent the site's politically correct policies; 2) Twitch's revamped Hateful Conduct and Harassment Policy was set to begin in two weeks. Twitch's new policy specifically aimed to protect women, LGBTQ folks, Black, Indigenous, and people of color who experience a disproportionate amount of harassment and abuse on the platform by banning certain emotes and words that target aforesaid groups (Twitch, 2020). In particular, Twitch's new policy bans certain terms, like simp, virgin, and incel, when used negatively and denying and removing emotes that are racist, sexist, or homophobic as they can be used in live-chats across Twitch. As an addendum, in April 2021, Twitch extended this policy further where if a streamer on Twitch engages in off-platform behavior, similar to Ryan Guiterrez, that incites harassment or threatens violence towards a particular group or person, then sanctions such as suspension and content removal will occur.

This presentation provides a discourse analysis through participant observation of Twitch's new hateful conduct and harassment policy in light of the U.S. Capitol insurrection and streamer behavior off-platform by focusing on user reactions. Indeed, after the U.S. Capitol insurrection, platforms, including Twitch, began banning white supremacist content and individuals. This presentation expands on extant scholarship on content moderation, platform governance, and affect theory by analyzing the blending of livestreaming platforms, streamer attitudes on and off platforms, and users' political concerns towards policy changes. Indeed, Tarleton Gillespie has noted that platforms are increasingly intervening in everyday life and shaping online communities and workers experiences (Gillespie, 2015, 2018). Furthermore, emotes or emojis act as digital pictograms that have reshaped digital communication in the public and corporate spheres (Stark and Crawford, 2015). However, Twitch's newly active role in streamers and emotes serve as the crux of dialogue on Twitch as well as a refiguring of livestreaming platforms as sites of contested political free and creative speech and behavior. Thus, this paper explores two intertwined questions: 1) What are the affective responses when livestreaming platform users think their free speech is being stymied? 2) How are livestreaming platforms mitigating harm when caused by their streamers and workers' political attitudes?

\section{Methods}

In order to answer the aforesaid research questions, I employ a discourse and audience analysis methodological approach through narrative inquiry of political attitudes and sentiments from Twitch users from January to April 2021. This presentation relies on preliminary data analysis coded using MAXQDA from popular press articles, scraping chats and using Twitch alongside ancillary platforms that Twitch users post on (Reddit, Discord, and Twitter) APIs to mine comments and channel replies, respectively, that contained titles or words mentioning: the new hateful conduct and harassment policy update, twitch banning simp, the confederate flag emote, Simp, Incel, Virgin, Trihard 
emote, or the PogChamp or Poggers emote. To organize my data, I used MAXQDA Data Management Software to aid in deploying an open and focused coding approach (Saladana and Omasta, 2018) of all screenshots, live streaming conversations, and scraped comments and threads. The author identified the following two theoretically informed themes of content.

\section{Emotes as (A)Political Discourse}

Emotes on Twitch and most livestreaming platforms are extremely popular modes of chatting and expressing various emotional responses to events in the stream. Emotes allow streamers and watchers to enact positive and negative emotional resonances that stick with one another sentimentally and generate communal contexts (Anable, 2018; Ahmed, 2012). With the banning of the infamous PogChamp emote alongside the revamped policy of new and current globally used emotes undergoing a review process, preliminary analysis signals user reactions were supportive of Twitch's policy to combat hate but not banning or reviewing individual emotes. Also, users sparingly noted Twitch's racist and oversexualized emotes, Trihard and custom Simp emotes, respectively, when commenting on the new policy. Indeed, Twitch users who also use Reddit, Discord, or Twitter concurred that emotes are not inherently racist or violent. Common sentiment fell along treating emotes as images, and thus apolitical, questioning if an emote could even be racist, and how specific people use emotes should be banned instead of the emote. The preliminary testimony here eclipses how emotes have become habitually part of political discourse and are now objects oriented towards politics. "We are not only directed towards objects," Sara Ahmed says, "but those objects also take us in a certain direction" (Ahmed, 2006, 545). Even though emotes may be images or objects of conversation, they indeed guide discourse on livestreaming platforms along with complex communal emotions.

\section{Simp Investigators \& Off-Platform Behavior}

Starting in April 2021, Twitch announced it would hire a third-party investigative law firm to aid in responding to reports of onsite and offsite streamer misconduct (Diaz, 2021). Alongside moderating users for using words like simp and incel alongside emotes coded to aforesaid words, Twitch would hold their streamers, who are often faces of popular, community-wide emotes, to a higher standard for engaging in harmful behavior beyond Twitch. Common user narratives in popular press comments or Reddit threads assert that this is merely corporations policing speech or Twitch trying to be politically correct rather than letting the justice system decide what qualifies as harassment. Narratives around harassment have often been aligned to put the burden of proof on the victim rather than holding the harasser accountable. Twitch is attempting to provide content moderation to remedy years of laissez-faire content moderation that impacted black streamers and users as well as governing dress codes that targeted women streamers. Indeed, Twitch is attempting to mitigate harmful political attitudes that have created a hostile environment on the platform and repair its reputation as a potentially hostile space for marginalized gamers to others off the platform.

\section{Conclusion}


Twitch's new hateful conduct and harassment policy aims to mitigate violent political attitudes on the platform. Bringing content moderation and affective theoretical frameworks in conversation with empirical, narrative data of user reactions to Twitch's new policy being enacted demonstrates how content moderation and platform governance for Twitch is shaping political and worker behavior on and off the platform. Additionally, this presentation addresses the affective narratives in response to how livestreaming platforms are curating the political expectations of etiquette and respectability on the internet.

\section{References}

Ahmed, S. (2006). Orientations: Toward a Queer Phenomeology. GLQ, 25-64. https://doi.org/10.2307/j.ctv125jk6w.5

Anable, A. (2018). Playing with feelings: video games and affect. University of Minnesota Press.

Diaz, A. (2021, April 7). Twitch will now use an 'investigative partner' for off-platform misconduct. Polygon. https://www.polygon.com/22372086/twitch-rulesharassment-streaming-community-guidelines-policy-hateful-conduct.

Gillespie, T. (2015). Platforms Intervene. Social Media + Society, 1(1), 205630511558047. https://doi.org/10.1177/2056305115580479

Gillespie, T. (2018). Custodians of the internet: platforms, content moderation, and the hidden decisions that shape social media. Yale University Press.

Introducing Our New Hateful Conduct \& Harassment Policy. Twitch Blog. (n.d.). https://blog.twitch.tv/en/2020/12/09/introducing-our-new-hateful-conductharassment-policy/.

Saldaña Johnny, \& Omasta, M. (2018). Qualitative research: analyzing life. SAGE Publishing.

Stark, L., \& Crawford, K. (2015). The Conservatism of Emoji: Work, Affect, and Communication. Social Media + Society, 1(2), 205630511560485.

https://doi.org/10.1177/2056305115604853 